\title{
LPS-induced systemic inflammation reveals an immunomodulatory role for the prion protein at the blood-brain interface
}

\author{
$\varnothing$. Salvesen, M. R. Reiten, A. Espenes, M. K. Bakkebø, M. A. Tranulis and C. Ersdal' (D)
}

\begin{abstract}
Background: The cellular prion protein $\left(\mathrm{Pr}^{\mathrm{C}}\right.$ ) is an evolutionary conserved protein abundantly expressed not only in the central nervous system but also peripherally including the immune system. A line of Norwegian dairy goats naturally devoid of $\operatorname{PrPC}^{C}$ (PRNP ${ }^{\text {Ter/Ter }}$ ) provides a novel model for studying $\operatorname{PrP}^{C}$ physiology.

Methods: In order to explore putative roles for $\mathrm{PrP}^{C}$ in acute inflammatory responses, we performed a lipopolysaccharide (LPS, Escherichia coli O26:B6) challenge of 16 goats (8 PRNP ${ }^{+/+}$and 8 PRNPTer/Ter) and included 10 saline-treated controls (5 of each PRNP genotype). Clinical examinations were performed continuously, and blood samples were collected throughout the trial. Genome-wide transcription profiles of the choroid plexus, which is at the blood-brain interface, and the hippocampus were analyzed by RNA sequencing, and the same tissues were histologically evaluated.

Results: All LPS-treated goats displayed clinical signs of sickness behavior, which were of significantly $(p<0.01)$ longer duration in animals without $\mathrm{PrP}^{\mathrm{C}}$. In the choroid plexus, a substantial alteration of the transcriptome and activation of Iba1-positive cells were observed. This response included genotype-dependent differential expression of several genes associated with the immune response, such as ISG15, CXCL12, CXCL14, and acute phase proteins, among others. Activation of cytokine-responsive genes was skewed towards a more profound type I interferon response, and a less obvious type II response, in $\mathrm{PrP}^{\mathrm{C}}$-deficient goats. The magnitude of gene expression in response to LPS was smaller in the hippocampus than in the choroid plexus. Resting state expression profiles revealed a few differences between the PRNP genotypes.
\end{abstract}

Conclusions: Our data suggest that $\operatorname{PrP}^{C}$ acts as a modulator of certain pathways of innate immunity signaling, particularly downstream of interferons, and probably contributes to protection of vulnerable tissues against inflammatory damage.

Keywords: Cellular prion protein, Systemic inflammation, Lipopolysaccharide (LPS), Innate immunity, Choroid plexus, Hippocampus, Transcriptome, Sickness behavior

\section{Background}

The cellular prion protein $\left(\operatorname{PrP}^{\mathrm{C}}\right)$ has been extensively studied for decades, but its normal function is still not fully understood. However, expression of this highly conserved protein across tissues in vertebrates suggests that it may have roles in a variety of physiological functions [1]. Accumulation of the misfolded isoform $\left(\mathrm{PrP}^{\mathrm{Sc}}\right)$ occurs in all prion disorders, and it has been postulated

* Correspondence: cecilie.ersdal@nmbu.no

Faculty of Veterinary Medicine, Norwegian University of Life Sciences, Sandnes, Norway that loss of $\operatorname{PrP}^{\mathrm{C}}$ function participates in the progression of these diseases [2]. Thus, identifying the normal function of $\mathrm{PrP}^{\mathrm{C}}$ is considered an essential step in understanding the pathogenesis of prion disorders.

$\operatorname{PrP}^{C}$ is abundantly expressed not only in the central nervous system (CNS) but also in non-neural tissues such as gonads, the pregnant uterus, and the immune system [3-5]. Several roles for $\operatorname{PrP}^{\mathrm{C}}$ in immunological processes have been suggested (reviewed in [6]). Lack of $\operatorname{PrP}^{C}$ seems to exacerbate inflammation, both in the periphery [7] and in the CNS [8], as well as ischemic 
[9-11] and traumatic [12] brain lesions. Likewise, $\operatorname{PrP}^{C}$ has been linked to regulation of pro- and antiinflammatory cytokines upon systemic lipopolysaccharide (LPS) challenge [13].

Systemic administration of LPS activates the Toll-like receptor 4 signaling cascade in a range of immune cells, resulting in synthesis and release of a variety of proinflammatory cytokines [14]. This, in turn, induces characteristic signs of sickness behavior, which includes depression, periods of shivering, and reduced appetite and locomotor activity [15]. We recently demonstrated that LPS is a potent activator of innate immunity in goats, describing a dynamic regulation of leukocyte genes involved in immunological processes [16]. Because only small amounts of LPS and cytokines cross the blood-brain barrier (BBB) [17], information from the periphery is transmitted to the CNS through neuronal and humoral communication routes. Pro-inflammatory cytokines and LPS can stimulate the vagus nerve, directly initiating afferent signaling to the brain [18]. The humoral route is characterized by circulating cytokines that activate endothelial cells of the BBB or act on tissues that lack BBB, such as the circumventricular organs and choroid plexus (ChP) [19]. Consequently, a mirror image of peripheral cytokines is created within the brain. The ChP is localized within the brain ventricular system and is composed of vascularized stroma surrounded by a monolayer of epithelial cells. The epithelial cells are responsible for the production of cerebrospinal fluid and can release cytokines into the ventricular system. Thus, the $\mathrm{ChP}$ plays a key role in transmitting signals into the brain during inflammatory conditions $[20,21]$. The cellular composition of the stroma can be dynamically altered through recruitment of circulating immune cells, such as lymphocytes, neutrophils, and monocytes $[22,23]$. Although the hippocampus is considered more immunoprivileged than the ChP, systemic LPS challenge may also impair hippocampal function [24, 25]. Certainly, cytokine receptors such as IL1R, which is fundamental in the response to inflammatory signals, are expressed in the hippocampus [26].

Recently, a nonsense mutation early in the gene encoding $\operatorname{PrP}^{C}(P R N P)$ in Norwegian dairy goats was discovered [27]. The mutation terminates $\operatorname{PrP}^{\mathrm{C}}$ synthesis only seven amino acids into the mature protein. Goats homozygous for the mutation $\left(P R N P^{\mathrm{Ter} / \mathrm{Ter}}\right)$ are devoid of $\operatorname{PrP}^{\mathrm{C}}$ and postulated to be scrapie-resistant $[27,28]$. Physiological and immunological studies have not identified major disturbances under normal herd conditions, which is in agreement with studies in transgenic animals without $\operatorname{PrP}^{\mathrm{C}}[29,30]$. However, closer phenotypic characterization indicates a small increase in red blood cell count of $\operatorname{PrP}^{\mathrm{C}}$-deficient goats compared both with normal animals and with goats heterozygous for the mutation [28]. These outbred, non-transgenic goats provide a new model for studying $\operatorname{PrP}^{\mathrm{C}}$ physiology.
We hypothesized that goats without $\mathrm{PrP}^{\mathrm{C}}$ are more susceptible to inflammation or stressful stimuli. To investigate this, we performed a longitudinal LPS study in normal $\left(P R N P^{+/+}\right)$and $\operatorname{PrP}^{\mathrm{C}}$-deficient goats $\left(P R N P^{\mathrm{Ter} / \mathrm{Ter}}\right)$ comprising clinical, biochemical, and hematological responses, as well as end-point tissue transcriptional profiles and characterization of morphological changes. In the current paper, we focus on the $\operatorname{Pr} \mathrm{P}^{\mathrm{C}}$-rich hippocampus, which is important in behavior and memory, as well as the ChP, an essential tissue in the interplay between the periphery and the brain.

\section{Methods}

\section{Animals}

A total of 26 Norwegian dairy goat kids, $13 P R N P^{\mathrm{Ter} / \mathrm{Ter}}$ and $13 \mathrm{PRNP}^{+/+}$animals, were included in the study. The goats were kept under a 16-h light/8-h dark cycle, housed in groups of two to four, and acclimatized at least 21 days before the experiment. Hay and water were provided ad libitum, and they were fed a commercial goat pellet concentrate. During the acclimatizing period, clinical examinations were performed three times, and fecal and blood samples were analyzed to ensure that the animals were healthy before the experiment. An overview of the study groups including treatment, animal number, age, weight, and gender can be found in Additional file 1a.

\section{LPS challenge}

The goats were split in groups as follows: 16 goats (8 PRNP $^{\mathrm{Ter} / \mathrm{Ter}}$ and $8 \mathrm{PRNP}^{+/+}$) that received LPS intravenously and a control group of 10 goats (5 $P R N P^{\text {Ter/Ter }}$ and $5 \mathrm{PRNP}^{+/+}$) that were given corresponding volumes of sterile saline. Based on existing literature $[31,32]$ and a pilot titration study (data not included), the LPS group received a dual dose of LPS (Escherichia coli O26:B6, L2654 Sigma-Aldrich, USA) with a 24-h time interval between doses; $0.1 \mu \mathrm{g} / \mathrm{kg}$ (day 1) and $0.05 \mu \mathrm{g} / \mathrm{kg}$ (day 2). As goats are very sensitive to LPS, the second dosage was reduced to avoid the risk of sensitization and mortalities. The animals were euthanized by an overdose of pentobarbital $5 \mathrm{~h}$ after the second LPS challenge. An overview of the study protocol is given in Additional file $1 \mathrm{~b}$.

\section{Clinical examination}

Clinical examination, including rectal temperature, heart and respiratory rate, and rumen contraction frequency was performed by veterinary surgeons at 12 time points during the first $7 \mathrm{~h}$ of day 1 and at 9 time points after the second LPS injection. Measurements of rectal temperature were repeated three times at each time point. Clinical examination was performed correspondingly, but at fewer time points, in control animals. 
The clinical examination and evaluation of sickness behavior were scored blinded with respect to genotype. Signs of sickness behavior were recorded by evaluating body position (standing, lying, head and ear position), locomotor activity, social interaction, appetite, and shivering. Based on this, goats were scored as presenting "sickness behavior" (S) or "no sickness behavior" (N) every $15 \mathrm{~min}$. The animals were evaluated until three consecutive "N" scorings were recorded, and the total duration of sickness behavior was calculated.

\section{Blood sampling, hematology and biochemistry}

Blood samples (EDTA and whole blood) were drawn from v. jugularis using a vacutainer system (BD Company, USA). Baseline samples $(0 \mathrm{~h})$ were taken within 30 min before LPS challenge. The other sampling times were $1,2,5$, and $24 \mathrm{~h}$ after the day 1 LPS administration. Hematology, including a complete blood count, was performed immediately by using the ADVIA 120 Hematology system (caprine analyzing program). Whole blood tubes were centrifuged, and serum stored at $-20{ }^{\circ} \mathrm{C}$ until biochemical analysis. Serum total protein, albumin, and glucose were analyzed by ABX Pentra 400 (Horiba, France) and ceruloplasmin by Cobas Mira Plus (Roche). Copper was quantified by AAnalyst 300 atomic absorption spectrometer (PerkinElmer, USA).

\section{Histological examination}

The left half of the brain was removed immediately from euthanized goats and immersion-fixed in $4 \%$ formaldehyde for 1 week. Defined brain slices were then dehydrated in graded ethanol and paraffin embedded. Morphological changes, including neuronal chromatolysis, single-cell necrosis, and inflammatory cell infiltration, were evaluated by analysis of hematoxylin and eosin-stained 4- $\mu \mathrm{m}$-thick tissue sections. Brain regions, including hippocampus, $\mathrm{ChP}$ in the lateral ventricle, and obex, were investigated.

\section{Immunohistochemistry and semi-quantitative scoring}

Paraffin sections ( $4 \mu \mathrm{m}$ thick) from the abovementioned areas were mounted on Superfrost ${ }^{\oplus}$ Plus slides (Menzel-Gläser, Thermo Scientific). The distribution and morphological appearance of the astrocyte marker, GFAP (Dako, Z0334), and the microglia/macrophage marker, Iba1 (Wako, 019-19741), were investigated by immunohistochemistry. The sections were dried overnight at $58{ }^{\circ} \mathrm{C}$, deparaffinized in xylene, and rehydrated through decreasing concentrations of graded ethanol. For Iba1 analysis, epitope retrieval was performed by trypsinization (10 mg/ml, 1:10 0.1 M Tris/HCl-buffer, $0.1 \% \mathrm{CaCl}_{2}$ ) for $30 \mathrm{~min}$ at $37{ }^{\circ} \mathrm{C}$. Endogenous peroxidase activity was blocked by incubation in $3 \% \mathrm{H}_{2} \mathrm{O}_{2}$ in methanol for $10 \mathrm{~min}$ at room temperature. The sections were then blocked in normal goat serum (1:50) diluted in $5 \%$ bovine serum albumin (BSA) for $20 \mathrm{~min}$ and incubated with the primary antibodies anti-Iba1 $(1.0 \mu \mathrm{g} / \mathrm{ml})$ or anti-GFAP $(1.9 \mu \mathrm{g} / \mathrm{ml})$ for $1 \mathrm{~h}$ at room temperature. Further steps were performed with EnVison+ kit (Dako, K4009). The sections were counterstained with hematoxylin for $40 \mathrm{~s}$. Washing between steps was in Tris-buffered saline (TBS). All runs included a negative control section where the primary antibody was replaced with $1 \%$ BSA/TBS.

The sections were examined by light microscopy and a blinded, semi-quantitative evaluation was performed by an investigator. The labeling intensity of the Iba1 and GFAP signals, the number of and localization of cells, and the appearance of primary and secondary processes were scored as follows: $0=$ minimal, $1=$ little, $2=$ moderate, 3 = strong, including half-step grading.

\section{RNA extraction, quality control, and pooling}

Tissue samples were collected from the right half of the brain within $15 \mathrm{~min}$ after euthanasia. The samples were dissected into small pieces, immediately immersed in RNAlater and stored at $-80{ }^{\circ} \mathrm{C}$. RNA extraction was carried out using RNeasy Lipid Tissue Mini Kit (Qiagen, 74804) according to the manufacturer's instruction. The isolated RNA was quantified at optical density $(\mathrm{OD})_{260}$, and purity was assessed by $\mathrm{OD}_{260 / 280}$ and $\mathrm{OD}_{260 / 230}$ absorbance readings with a DeNovix DS-11 spectrophotometer (Wilmington, USA). RNA integrity was assessed by RNA 600 Nano chips in compliance with the Agilent Bioanalyzer 2100 system in all individual samples before pooling. RNA quality data are summarized in Additional file 1c.

Extracted RNA was diluted to $500 \mathrm{ng} / \mu \mathrm{l}$ and then remeasured three times. Equal amounts (ng) of RNA from individual samples were pooled, reaching a final amount of $15,000 \mathrm{ng}$. The samples were pooled according to tissue, treatment, and genotype making a total of eight pools. RNA samples were shipped on dry ice to Novogene (Hong Kong) for RNA sequencing. As the transcriptome profile might be sensitive to gender, one buck (LPS, $P R N P^{\text {Ter/Ter }}$ was excluded from the material, leaving only female samples.

\section{RNA sequencing}

After quality control, messenger RNA (mRNA) was enriched using oligo ( $\mathrm{dT}$ ) beads and then randomly fragmented. First-strand complementary DNA (cDNA) was synthesized using random hexamers and reverse transcriptase. Second-strand synthesis was done by nicktranslation using a buffer containing dNTPs, RNase $\mathrm{H}$, and $E$. coli polymerase I (Illumina). The cDNA fragments were processed using an end-repair reaction after the addition of a single "A" base, followed by adapter ligation. These products were then purified and amplified using 
PCR to generate the final cDNA library. The quality of each library was evaluated by 2100 Bioanalyzer (Agilent), followed by paired-end 150-bp sequencing on an Illumina HiSeq2000. The quality control summary can be found in Additional file $1 \mathrm{~d}$.

\section{Differential expression analysis}

Raw reads (FASTQ) were clipped and trimmed of adapter contamination, and those of low quality were removed. Quality-controlled FASTQ files were mapped to the Capra hircus (domestic goat) reference genome using the TopHat2 (v2.0.12) software with two mismatches. Mapping status is summarized in Additional file 1e. Differential gene expression analysis (DEA) was performed using DEGSeq2 (1.12.0) with the following criteria: $\log 2$ ratio \pm 0.59 (fold change \pm 1.5 ) and a false discovery rate (FDR) adjusted $q$-value $(q<0.05)$. For each tissue, four DEAs were performed. Differences in basal transcriptome levels were assessed by comparing $P R N P^{\mathrm{Ter} / \mathrm{Ter}}$ (saline) to $P R N P^{+/+}$ (saline). The genomic response to LPS in each genotype was assessed by comparing the LPS groups with the salinetreated control of the matching genotype. Finally, DEAs between $P R N P^{\text {Ter/Ter }}$ (LPS) and $P R N P^{+/+}$(LPS) were performed to identify differences between the genotypes during acute inflammation. FPKM (fragments per kilobase of exon per million fragments mapped) values, which take into account the effects of both sequencing depth and gene length, were used to estimate gene expression levels. Genes encoding ribosomal subunit proteins are not included in the tables.

\section{Gene ontology enrichment analysis}

To characterize the overall LPS effect, gene ontology (GO) analysis was performed on genes that were differentially expressed (DEGs) in at least one of the PRNP genotypes. We used the online PANTHER classification system to identify over-represented biological processes among the DEGs $[33,34]$. Because the C. hircus genome was not available, and the Bos taurus genome resulted in fewer mapped genes, the well-annotated Homo sapiens genome was used as reference. The fold enrichment displays the over-representation of genes in a given biological process, compared with the expected number in the reference genome. $p$ values $<0.05$ represents a statistical significant over-representation and are calculated by the binomial test as described in [33]. In total, eight genes (SAA3, OAS1L, MHCI, IFI203, VCAM, ADGRG6, $C 4$, and C21H14orf132) were not mapped to the GO reference genome.

\section{Validation of RNA sequencing by qPCR}

First, 600 ng total RNA from each individual sample was converted into first-strand cDNA using QuantiTect Reverse Transcription Kit (Qiagen, Germany) according to the manufacturer's instructions. A non-reverse transcriptase control (NoRT) and no template control (NTC) were included.

The expression of PRNP, IFI6, CXCL10, and SAA3 genes was investigated by Light cycler 480 qPCR using SYBR Green PCR Master Mix under the following conditions: initial denaturation for $5 \mathrm{~min}$ at $95{ }^{\circ} \mathrm{C}$, followed by 40 amplification cycles $\left(10 \mathrm{~s}\right.$ at $95{ }^{\circ} \mathrm{C}, 10 \mathrm{~s}$ at $60{ }^{\circ} \mathrm{C}$, and $15 \mathrm{~s}$ at $72{ }^{\circ} \mathrm{C}$ ) and construction of melting curves. For each primer assay, a pool of cDNA samples was used to make three separate series with the following dilutions: 1:2, 1:10, 1:50, 1:250, and 1:1250. Standard curves were constructed to obtain primer amplification efficiencies, correlations, and dynamic range. Internal normalization was performed against the $A C T B$ reference gene, and relative expression was calculated using the $2^{-\Delta \Delta \mathrm{Cq}}$ method [35]. Primer sequences are given in Additional file 1f.

\section{Descriptive and statistical analyses}

Clinical, biochemical, hematological, and qPCR expression data are presented as mean \pm standard error of the mean (SEM). Graphical and statistical analyses were performed in GraphPad Prism 6 (GraphPad software Inc., USA) and Microsoft Excel 2013. Comparisons between two groups were performed using Student's $t$ test, assuming equal variance.

\section{Results}

Prolonged sickness behavior in goats devoid of PrPC

Within the first 2 hours after LPS administration, all 16 goats displayed characteristic signs of sickness behavior, such as lowered head, hanging ears, and periods of shivering, as well as reduced social interaction, appetite, and locomotor activity. The mean duration of sickness behavior was significantly $(p<0.01)$ longer in the $P R N P^{\mathrm{Ter} / \mathrm{Ter}}$ group $(6.03 \pm 0.59 \mathrm{~h})$, than in the $P R N P^{+/+}$goats $(4.16 \pm$ $0.33 \mathrm{~h})$ after day 1 LPS challenge $(0.1 \mu \mathrm{g} / \mathrm{kg})$. The second LPS injection $(0.05 \mu \mathrm{g} / \mathrm{kg})$ induced a significantly shorter period of sickness behavior compared with day 1 , but the difference between the genotypes was non-significant (Fig. 1a, b). $P R N P^{\text {Ter/Ter }}$ goats displayed a slightly higher mean body temperature at all time points throughout the experiment. The fever response was biphasic, with the lower peak at $1.5 \mathrm{~h}$ and the highest temperature at $3.5 \mathrm{~h}$ on day 1 . A quick onset monophasic fever, peaking at $1.5 \mathrm{~h}$ was measured on day 2 (Fig. 1c). Tachycardia was observed in both genotypes, reaching a maximum at $5 \mathrm{~h}$ post challenge (data not shown).

The number of white blood cells declined after LPS challenge (Fig. 1d), abruptly in neutrophils and monocytes and more gradually in lymphocytes, with no differences between the genotypes (Additional file 2a). This contrasts with what has been reported in Prnp-knock out (KO) mice following systemic LPS injection [13], and 


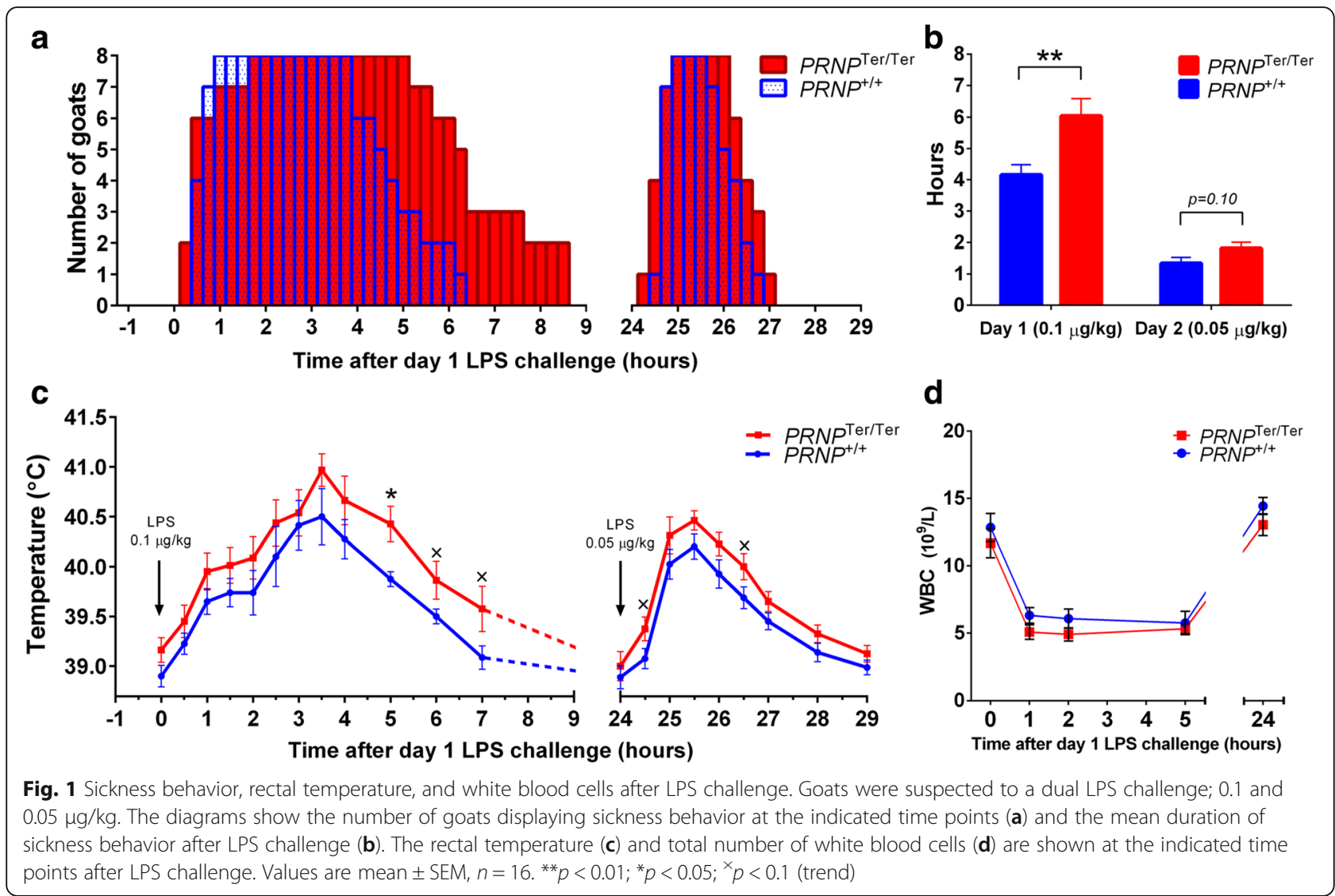

our data do not support a role for $\operatorname{PrP}^{\mathrm{C}}$ in leukocyte extravasation and recovery. Total serum protein decreased in both groups, and the level of albumin was significantly lower in PRNP $P^{\text {Ter/Ter }}$ animals at 5 and $24 \mathrm{~h}$. No differences were observed in serum ceruloplasmin or copper. Although impaired glucose homeostasis has been observed in Prnp-KO mice [36], blood glucose was regulated similarly between the two genotypes after LPS challenge (Additional file 2b). None of the control animals displayed alterations in clinical, hematological, or biochemical parameters in response to saline injection and handling stress.

Systemic LPS challenge induces substantial alterations in the choroid plexus transcriptome and reveals differences between PRNP genotypes

In the ChP, 92 genes were upregulated and 25 genes downregulated in at least one PRNP genotype upon LPS challenge (Fig. 2). Eighty-seven percent of the DEGs were regulated in the same direction in both PRNP genotypes, but some had a $\log 2$ ratio or $q$-value outside our filtration criteria (Additional file 3). GO enrichment analysis of upregulated genes displayed an over-representation of genes involved in type I interferon (IFN) signaling, collagen catabolism, and leukocyte migration. Additionally, 19 genes were characterized as being involved in the innate immune response and 23 genes were cytokine-responsive (Additional file 4a).

Finally, we identified 25 differentially expressed genes between the two genotypes after LPS treatment, which included several immune genes, as well as genes involved in extracellular matrix stability and oxidative phosphorylation (Table 1). Given the previous report on cytokines being influenced by $\operatorname{PrP}^{C}$ [13], we analyzed the 23 genes characterized as cytokine-responsive (GO:0034097) with less stringent criteria (fold change \pm 1.2 and $p<0.05$ ). Comparing the $\log 2$ ratio of the these genes after LPS administration, a relatively higher type I IFN response, and less prominent type II IFN response, was noted in the $\operatorname{PrP}^{\mathrm{C}}$-deficient goats (Fig. 3).

\section{Systemic LPS challenge induces minor alterations in hippocampus transcriptome in both PRNP genotypes} According to the filtration criteria, only 10 genes were upregulated and 3 genes downregulated, in one or both PRNP genotypes after LPS challenge (Fig. 2 and Table 2). Five of the upregulated genes (MT2, CXCL9, CXCL10, TGM2, and IFI6) were classified as being involved in cytokine signaling (Additional file 4b). CXCL10, CXCL9, and TGM2 were significantly upregulated in both genotypes, whereas IFI6 was only upregulated in normal goats. The expression of IFI6, however, was already high 

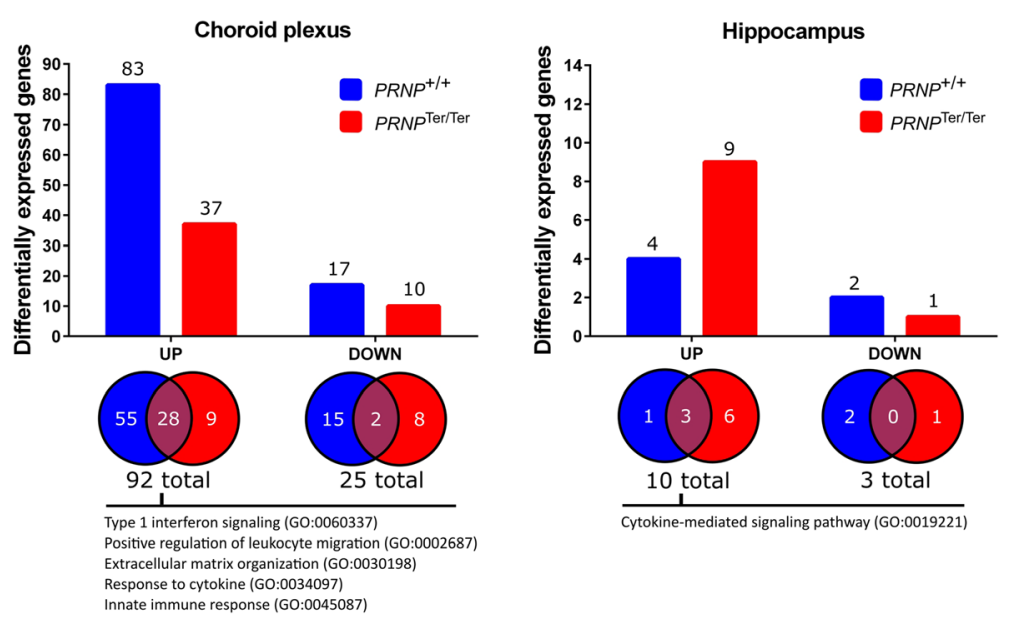

Fig. 2 Upregulated and downregulated genes in the choroid plexus and hippocampus after LPS challenge. Differential expression analyses were performed by comparing the LPS groups to the saline-treated control group of the matching genotype (log2 ratio \pm 0.59 and $q<0.05$ ). Venn diagrams show genes that overlap in the two genotypes. The top over-represented biological functions (GO terms) are included for upregulated genes. LPS, $n=15$. Saline, $n=10$

at rest in $P R N P^{\mathrm{Ter} / \mathrm{Ter}}$ goats (saline group). Six genes (ATP5I, GFAP, HOPX, MT2, MT1A, and SLC14A1) were significantly upregulated in the $\operatorname{PrP}^{\mathrm{C}}$-deficient goats and slightly, but non-significantly, upregulated in the normal goats. In the LPS-treated normal goats, a slight increase in PRNP expression was observed by RNA sequencing and $\mathrm{qPCR}$, but this was not statistically significant.

\section{Transcriptome analyses of choroid plexus and hippocampus at rest (saline) reveal minor differences between the PRNP genotypes}

The basal expression was investigated by a differential expression analysis of the saline-treated groups, and only minor differences were observed. $P R N P^{\text {Ter/Ter }}$ goats displayed higher expression of IFI6 in both hippocampus and in the ChP. DKK3,CHGA, and MYOM2 were slightly upregulated in the $\operatorname{PrP}^{\mathrm{C}}$-deficient goats, whereas the transcript levels of $P R N P$ were decreased with a $\log 2$ ratio of -4.4 in the hippocampus and -2.3 in the ChP. However, as RNA was extracted from tissues as a whole, the dilution effect might mask more subtle phenotypes related to loss of $\operatorname{PrP}^{C}$ in certain cell populations.

\section{Systemic LPS activates Iba1-positive cells in the choroid plexus and astrocytes in the hippocampus in both PRNP genotypes}

In the ChP, expression of the Iba1-encoding allograft inflammatory factor 1 (AIF1) and of the microglia/ macrophage phenotype activation markers was increased (Fig. 4a, b). This corresponded with increased signal and number of Iba1-positive cells (Fig. 4c, d). These cells were primarily located at the basal side of the epithelial cells and within the stroma, with processes extending between the epithelium and around blood vessels. Some
Iba1-positive cells were presumably migrating towards the apical surface and found between the epithelial cells and at the apical surface. No GFAP-labeling was observed in the ChP, confirming that this tissue does not contain astrocytes. Evaluation of the HE-stained sections did not reveal infiltration of inflammatory cells within the stroma (Fig. 4e), but an increased number of leukocytes was observed in the blood vessels (leukostasis).

In the hippocampus, LPS treatment upregulated GFAP expression, and increased GFAP signal was detected by immunohistochemistry in the molecular layer, subgranular layer, and hilus (Fig. 5). Activated astrocytes had more distinct primary and secondary processes, than seen in saline controls. Morphological evaluation of Iba1-stained microglia did not identify any effect of treatment or genotype in the hippocampus or obex. Some individuals had a few single-cell necrosis in the granular and subgranular layer, but this was not related to genotype or treatment. No evidence of disruption of the $\mathrm{BBB}$ was observed by light microscopy.

\section{Validation of RNA sequencing by qPCR on individual RNA samples}

Four target genes (PRNP, IFI6, CXCL10, and SAA3) were investigated by $\mathrm{qPCR}$ on individual RNA samples from both tissues. A strong correlation $(r=0.989$, $p<0.0001$, Pearson correlation) was observed between expression values of RNAseq and qPCR, and differential expression was confirmed in 26 out of 28 comparisons. The increased IFI6 expression in PRNP $P^{\mathrm{Ter} /}$ Ter goats at rest was primarily due to large biological variability. In the hippocampus, $S A A 3$ was not detected by RNAseq in saline groups, and comparison 
Table 1 Choroid plexus DEGs between PRNP genotypes after LPS treatment

\begin{tabular}{|c|c|c|c|c|}
\hline Gene ID & Symbol & Gene name & Log2 ratio & Top functions \\
\hline 100860813 & $\mathrm{MHC} \|$ & HA25 & 1.80 & Antigen presentation (extracellular pathway) \\
\hline 102178155 & FTSJ1 & FtsJ RNA methyltransferase homolog 1 & 1.53 & Methyltransferase \\
\hline 102169982 & ISG15 & ISG15 ubiquitin-like modifier & 0.99 & ISGylation, innate immunity \\
\hline 102189650 & ANK3 & Ankyrin 3 & 0.75 & Cell motility, activation, proliferation, \\
\hline 102187800 & UBB & Ubiquitin B & 0.73 & Targeting of proteins for degradation \\
\hline 102184593 & SPOCK2 & SPARC/osteonectin, cwCV and kazal-like domains proteoglycan 2 & 0.68 & Extracellular matrix structure \\
\hline 102183219 & BGN & Biglycan & -0.60 & Extracellular matrix structure, innate immunity \\
\hline 102188061 & ATP5E & $\begin{array}{l}\text { ATP synthase, } \mathrm{H}+\text { transporting, mitochondrial F1 complex, } \\
\text { epsilon subunit }\end{array}$ & -0.63 & Catalyzes ATP synthesis, oxidative phosphorylation \\
\hline 102185420 & COL24A1 & Collagen type XXIV alpha 1 chain & -0.66 & Extracellular matrix structure \\
\hline 102172487 & $\mathrm{CP}$ & Ceruloplasmin & -0.66 & Acute phase protein, ferroxidase enzyme \\
\hline 102169556 & CXCL12 & C-X-C motif chemokine ligand 12 & -0.67 & Chemoattractant, innate immunity \\
\hline 102172637 & LECT1 & Leukocyte cell derived chemotaxin 1 & -0.69 & $\begin{array}{l}\text { Promotes chondrocyte growth, inhibit } \\
\text { angiogenesis }\end{array}$ \\
\hline 102170107 & ATP5J2 & $\begin{array}{l}\text { ATP synthase, } \mathrm{H}+\text { transporting, mitochondrial Fo complex } \\
\text { subunit F2 }\end{array}$ & -0.70 & Catalyzes ATP synthesis, oxidative phosphorylation \\
\hline 102181355 & PCOLCE & Procollagen C-endopeptidase enhancer & -0.75 & Collagen precursor peptidase activator \\
\hline 100860756 & OGN & Osteoglycin & -0.76 & Growth factor activity \\
\hline 102179198 & TNC & Tenascin C & -0.81 & Extracellular matrix structure \\
\hline 102182694 & CXCL14 & C-X-C motif chemokine ligand 14 & -0.81 & Chemoattractant, immunomodulatory \\
\hline 102177419 & NDUFA1 & NADH:ubiquinone oxidoreductase subunit A1 & -0.85 & Component of the respiratory chain, mitochondria \\
\hline 102191086 & $\mathrm{COCH}$ & Cochlin & -1.03 & $\begin{array}{l}\text { Extracellular matrix, pro-inflammatory, cytokine } \\
\text { regulatory }\end{array}$ \\
\hline 102187830 & COL17A1 & Collagen type XVII alpha 1 chain & -1.04 & Hemidesmosome component \\
\hline 102189939 & ATP5I & $\begin{array}{l}\text { ATP synthase, } \mathrm{H}+\text { transporting, mitochondrial Fo complex } \\
\text { subunit } \mathrm{E}\end{array}$ & -1.58 & $\begin{array}{l}\text { Catalyze ATP synthesis, oxidative } \\
\text { phosphorylation }\end{array}$ \\
\hline 100860915 & ASIP & Agouti signaling protein & -2.14 & Paracrine signaling, pigmentation \\
\hline 102180584 & $\mathrm{MHCl}$ & BOLA class I histocompatibility antigen, alpha chain BL3-7 & -2.32 & Antigen presentation (cytosolic pathway) \\
\hline 102169975 & PRNP & Prion protein & -2.80 & Unknown, cytoprotective \\
\hline 102176354 & $\mathrm{HP}$ & Haptoglobin & -3.25 & Acute phase protein, bind hemoglobin \\
\hline
\end{tabular}

Differential expression analysis was performed by comparing LPS-treated $P R N P^{\text {Ter/Ter }}$ vs. $P R N P^{+/+}(\log 2$ ratio \pm 0.59 and $q<0.05) . n=15$

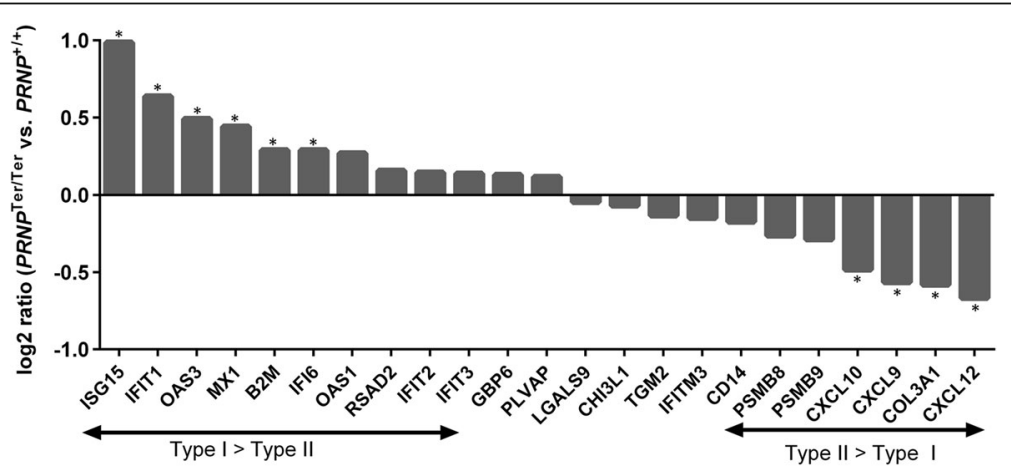

Fig. 3 Comparison of ChP cytokine-responsive genes (GO:0034097) after LPS challenge between genotypes. Expression levels were investigated by transcriptome analysis (Illumina HiSeq 2000) on RNA extracted from the choroid plexus. Transcripts of genes stimulated primarily by type I interferons increased in PrPC -deficient goats compared with $P R N P^{+/+}$goats, whereas genes stimulated primarily by type II interferons were reduced. ${ }^{*}$ Fold change \pm 1.2 and $p<0.05 . n=15$ 
Table 2 Upregulated and downregulated genes in the hippocampus after LPS challenge

\begin{tabular}{|c|c|c|c|c|c|}
\hline \multirow[b]{2}{*}{ Gene ID } & \multirow[b]{2}{*}{ Symbol } & \multirow[b]{2}{*}{ Gene name } & \multicolumn{2}{|c|}{ Log2 ratio, LPS vs. saline } & \multirow[b]{2}{*}{ Top functions } \\
\hline & & & PRNP ${ }^{+/+}$ & PRNPTer/Ter & \\
\hline 102185230 & IFI6 & Interferon alpha inducible protein 6 & $1.19^{*}$ & -0.05 & Regulation of apoptosis \\
\hline 100860873 & CXCL10 & $\mathrm{C}-\mathrm{X}-\mathrm{C}$ motif chemokine ligand 10 & $3.37^{*}$ & $3.07^{*}$ & Chemoattractant \\
\hline 102187851 & CXCL9 & C-X-C motif chemokine ligand 9 & $2.93^{*}$ & $2.65^{*}$ & Chemoattractant \\
\hline 102185477 & TGM2 & Transglutaminase 2 & $1.25^{*}$ & $1.36^{*}$ & Unclear, involved in phagocytosis \\
\hline 102189939 & ATP5I & $\begin{array}{l}\text { ATP synthase, } \mathrm{H}+\text { transporting, mitochondrial Fo } \\
\text { complex subunit } \mathrm{E}\end{array}$ & 0.53 & $0.76^{*}$ & Catalyze ATP synthesis \\
\hline 102190069 & GFAP & Glial fibrillary acidic protein & 0.34 & $0.62^{*}$ & Cell communication, mitosis, BBB function \\
\hline 102178715 & HOPX & HOP homeobox & 0.35 & $0.65^{*}$ & Unknown \\
\hline 102188072 & MT2 & Metallothionein-2 & 0.29 & $0.91^{*}$ & Metal-binding, neuroprotection \\
\hline 102188618 & MT1A & Metallothionein-1A & 0.58 & $1.40^{*}$ & Metal-binding, neuroprotection \\
\hline 100860878 & SLC14A1 & Solute carrier family 14 member 1 & 0.54 & $1.59^{*}$ & Membrane transport (urea) \\
\hline 102186073 & AQP4 & Aquaporin 4 & $-0.61^{*}$ & -0.31 & Membrane transport (water) \\
\hline 102175716 & MYO10 & Myosin X & $-0.66^{*}$ & -0.53 & Motor molecule, bind actin \\
\hline 102186825 & COL9A2 & Collagen type IX alpha 2 chain & -0.43 & $-0.64^{*}$ & Extracellular matrix structure \\
\hline
\end{tabular}

Differential expression analyses were performed by comparing the LPS groups to the saline control group of the matching genotype. Italic values indicate a true differential expression. LPS, $n=15$. Saline, $n=10$

* $\log 2$ ratio $\pm 0.59, q<0.05$
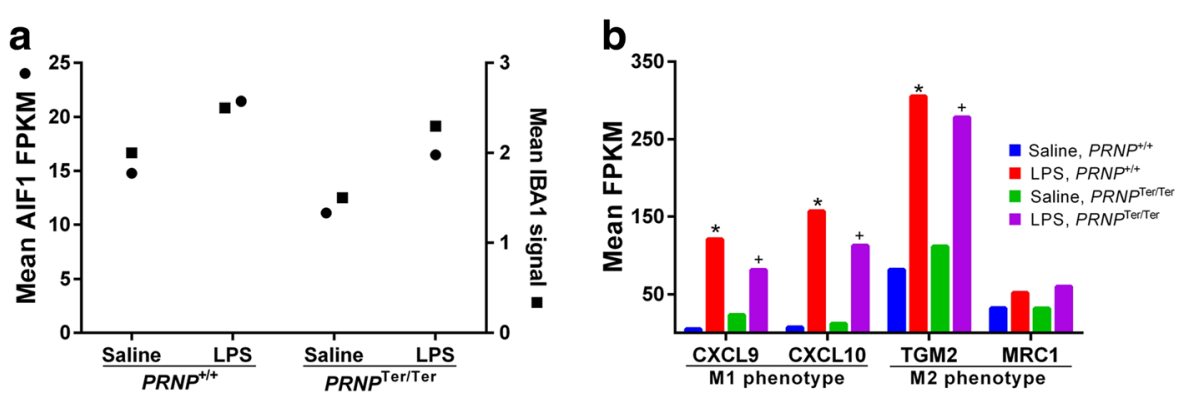

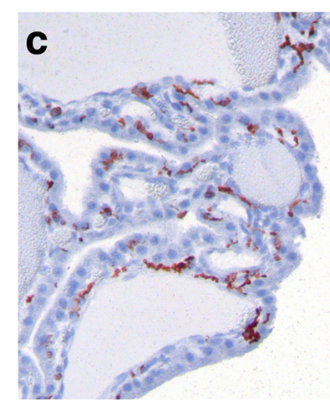

Saline, Iba1

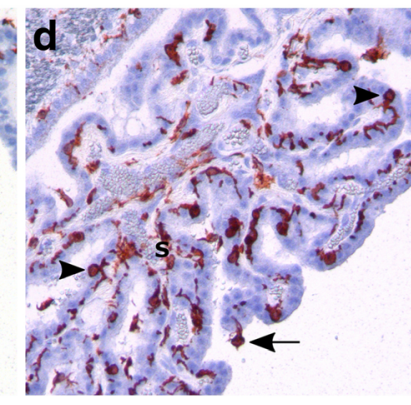

LPS, Iba1

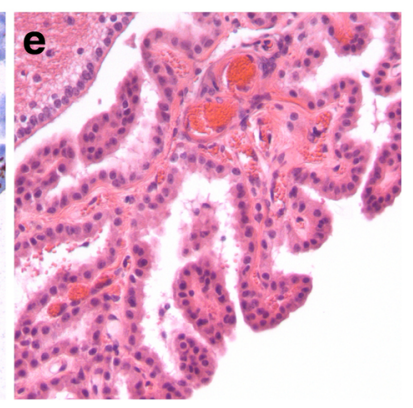

LPS, HE

Fig. 4 LPS upregulates AIF1/IBA1 and macrophage/microglia phenotype markers in the choroid plexus. Expression levels were investigated by transcriptome analysis (IIlumina HiSeq 2000) on RNA extracted from the choroid plexus. Allograft inflammatory factor 1 (AIF1) expression corresponded with increased IBA1 signal detected by immunohistochemistry (a). Genes indicating activation of macrophage/microglia phenotype $\mathrm{M} 1$ and M2 increased in both PRNP genotypes. A significant upregulation (log2 ratio $>0.59, q<0.05$ ) in $P R N P^{+/+}$and $P R N P^{T e r / T e r}$ goats is indicated by an asterisk and plus sign, respectively (b). Some Iba1-positive cells are present in the choroid plexus of saline-treated animals (c). The number of Iba1-positive cells is increased, and the cells have a different location and longer processes in LPS-treated animals. Cells are localized within the stroma (S), between the epithelial cells (arrowhead) and protruding from the apical surface (arrow) (d). No infiltration of inflammatory cells is observed within the stroma (e). $\times 200$ magnification. LPS, $n=16$. Saline, $n=10$ 


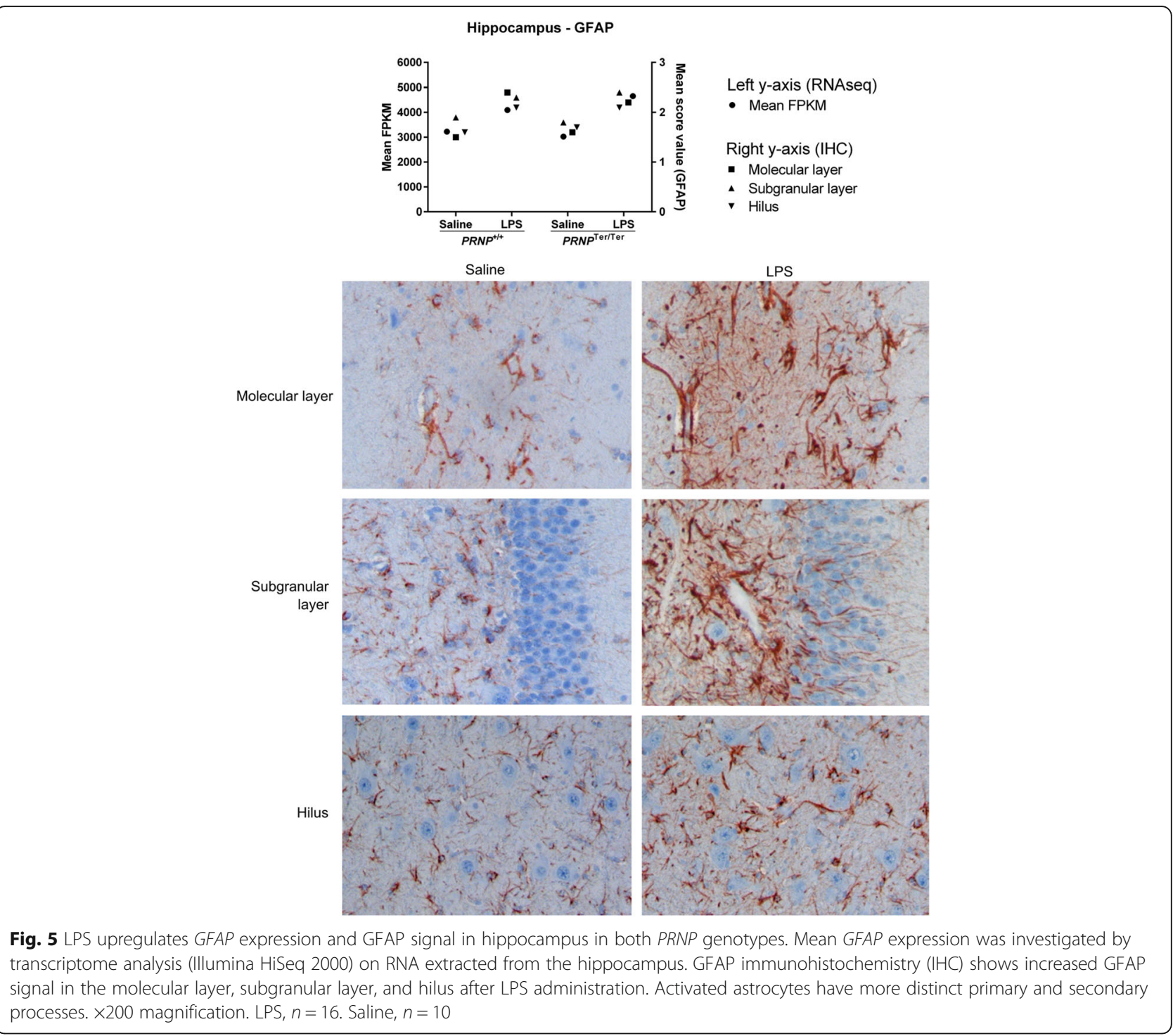

with relative qPCR expression was not possible (Additional file 5).

\section{Discussion}

The high degree of conservation of the PRNP gene across species [37] suggests that the protein possesses important biological functions. These have, however, proven difficult to pin-point even after the creation of Prnp-KO mice [38]. It has been proposed that compensatory mechanisms could mask loss-of-function phenotypes under normal conditions and become evident during stress such as inflammation. For instance, Prnp-KO mice displayed an exacerbated disease progression of experimental autoimmune encephalomyelitis [8] and colitis [7]. Here, we report the first study of the inflammatory response in a unique, non-rodent model naturally devoid of $\operatorname{PrP}^{\mathrm{C}}$. Considering the high PRNP expression in the hippocampus and the role of the choroid plexus (ChP) in responding to inflammatory signals at the blood-brain boundary, we investigated both these tissues by full-scale transcriptome analysis. Our data suggest a role for $\operatorname{PrP}^{C}$ in modulating the innate immune response.

Systemic LPS challenge induced characteristic signs of sickness behavior that was prolonged by about $2 \mathrm{~h}$ in $P R N P^{\text {Ter/Ter }}$ goats after the initial high dose of LPS $(0.1 \mu \mathrm{g} / \mathrm{kg})$. This is a novel clinical loss-of-function phenotype, pointing to a more potent inflammatory response in the absence of $\operatorname{PrP}^{\mathrm{C}}$. When the dosage was halved on day 2 , the mean duration of sickness behavior was only about $1-2 \mathrm{~h}$ in both groups. The difference between genotypes was similar as day 1 , but not statistically significant $(p=0.1)$. This suggests that the lower dose of LPS did not induce a sufficient amount of inflammatory stress to clearly separate the two genotypes. 
In the ChP, a clear acute phase response as well as activation of a range of interferon-stimulated genes was observed, which underlines the widespread role of these genes in the host defense to bacterial endotoxin [16]. Interestingly, several genes associated with the immune response were differentially expressed between the PRNP genotypes after LPS challenge. This included acute phase proteins genes and multiple chemokines, as well as $\mathrm{COCH}$ that has an anti-bacterial role by regulating local cytokine production [39]. Based on previous reports of $\operatorname{PrP}^{\mathrm{C}}$ regulating cytokines [13], we compared the 23 genes characterized as cytokine-responsive (GO:0034097) between the two genotypes. Most of these genes are primarily induced by type I and/or type II IFNs [40, 41]. Notably, there was a relatively more pronounced type I IFN response and a weaker type II response in $\operatorname{PrP}^{\mathrm{C}}$-deficient goats, compared with the normal group. A potential role of $\operatorname{PrP}^{\mathrm{C}}$ in regulating type II IFN response has been previously suggested, as IFN- $\gamma$ levels were decreased in ConA-treated PrP $0 / 0$ splenocytes [42], but type I interferon signaling has received less attention. Type I interferons are key modulators of innate immunity and may affect the manifestation of sickness behavior by facilitating the immune activation of other cytokines [43]. Indeed, interferon signaling is involved in many of the effects previously attributed $\operatorname{PrP}^{\mathrm{C}}$, such as apoptosis [44], protection against oxidative stress [45], DNA repair [46], and depressive-like behavior immediately after stress [47, 48]. Taken together, our data indicate that $\operatorname{PrP}^{C}$ contributes as a modulator of innate immunity signaling, particularly downstream of type I interferons, which might affect the duration of sickness behavior.

The substantial activation of the ChP transcriptome, including upregulation of AIF1 expressed by activated macrophages/microglia, corresponded with a parallel increase in Iba1 signal. Markers of classical activation M1 (CXCL9, CXCL10) and alternative activation M2 (TGM2, MRC1) increased $[49,50]$, suggesting a combination of M1 and M2 phenotype of activated macrophages/Iba1-positive cells. Moreover, cytokines involved in leukocyte migration were upregulated, as well as genes involved in collagen catabolism and extracellular matrix organization, indicating that the integrity of the blood-cerebrospinal fluid barrier was altered. These findings agree with the observation of increased numbers of Iba1-positive cells in the ChP stroma, some of which were presumably migrating through the epithelium. The stromal cells could represent antigen-presenting cells as dendritic cells [51], recently blood-derived monocytes, or residing macrophages [52].

Not surprisingly, alterations in the hippocampus transcriptome were modest compared with those observed in the ChP, yet a somewhat similar cytokine response was present. However, it is possible that the filtration of single genes strictly by fold change and $q$-value might exclude biologically relevant pathways characterized by a subtle increase in a subset of genes. The two most upregulated genes in the hippocampus were CXCL9 and CXCL10, which are primarily induced by IFN- $\gamma$ signaling [41]. Recently, CXCL10 expression in hippocampus was traced to activated astrocytes and cells lining the blood vessels [53]. This suggests that endothelial cells within the $\mathrm{BBB}$, as well as nearby glial cells, react to circulating LPS and cytokines by releasing IFN- $\gamma$, which, in turn, stimulates expression of CXCL9 and CXCL10. Despite the important role of these chemokines in recruiting immune cells into the brain [54], no inflammatory cell infiltration was observed in our study. Although the overall LPS response in the hippocampus was similar in the two PRNP genotypes, two metallothioneins (MT) were significantly upregulated in $\operatorname{PrP}^{\mathrm{C}}$-deficient goats. Metallothioneins bind metals and scavenge free radicals and participate in reducing the inflammatory and oxidative stress [55]. In the brain, MT-I and MT-II are primarily expressed by activated astrocytes [55]. We further found that GFAP transcription increased significantly in $P R N P^{\mathrm{Ter} / \mathrm{Ter}}$ goats, indicating an early activation of astrocytes as previously described $[53,56]$. This was confirmed by an increased GFAP labeling after LPS treatment. Given the role of MTs [55] and $\operatorname{PrP}^{\mathrm{C}}$ [57] in neuroprotection, it is tempting to speculate that upregulation of metallothioneins in astrocytes could be part of a compensatory mechanism in goats devoid of $\operatorname{PrP}^{\mathrm{C}}$.

Systemic administration of LPS has been shown to activate microglia in the hypothalamus, thalamus, and brainstem as early as $8-24 \mathrm{~h}$ after LPS challenge [58], but murine hippocampal microglia were not activated until $48 \mathrm{~h}$ post challenge [59]. The latter study is consistent with our results as we did not observe increased AIF1 expression or altered Iba1 immunohistochemical labeling in hippocampus $29 \mathrm{~h}$ after the first LPS injection. Altogether, the transcriptional and morphological findings indicate that only a modest inflammation, with a predominance of astrocytes, was present in the hippocampus. This might not be sufficient to manifest clearly potential phenotypes related to the loss of $\mathrm{PrP}^{\mathrm{C}}$ and further suggests that this brain region is relatively protected from circulating endotoxins. Still, the clinical signs of sickness behavior, and difference between the PRNP genotypes in this respect, demonstrate the sensitivity of the CNS towards inflammatory insult and that this sensitivity is increased in the absence of $\operatorname{PrP}^{\mathrm{C}}$.

Although not statistically significant, LPS upregulated PRNP transcripts in both the hippocampus and ChP of $P R N P^{+/+}$goats, indicating a role for $\mathrm{PrP}^{\mathrm{C}}$ in acute inflammation. Similarly, systemic LPS upregulated $\operatorname{PrP}^{\mathrm{C}}$ in circulating neutrophils [60], whereas LPS incubation 
increased PRNP expression in neuronal cell cultures [61]. As expected, PRNP expression was low in $\operatorname{PrP}^{\mathrm{C}}$ deficient goats, regardless of treatment, which probably reflects nonsense-mediated mRNA decay [62].

\section{Conclusions}

This is the first report of an endotoxin challenge in a non-transgenic goat model naturally devoid of $\operatorname{PrP}^{\mathrm{C}}$. Animals without $\operatorname{Pr} \mathrm{P}^{\mathrm{C}}$ suffered a significantly prolonged period of sickness behavior after LPS challenge. Transcriptome data revealed that in the absence of $\operatorname{PrP}{ }^{\mathrm{C}}$, LPS induced an increased expression of a number of genes downstream of type I interferons. These results, together with the finding that PRNP was slightly upregulated upon LPS stimulation in normal goats, point to an immunomodulatory role for $\operatorname{PrP}^{\mathrm{C}}$ during inflammation. Importantly, a huge number of proteins contribute to modulating inflammatory responses, balancing pro- and anti-inflammatory signaling. This balancing act is vitally important for the organism and not dependent upon a few proteins or signaling pathways. Considering the many crossroads between innate immunity signaling and various aspects of cellular homeostasis, such as apoptosis and DNA repair, our data contribute to a new understanding of cellular functions previously ascribed to $\operatorname{PrP}^{\mathrm{C}}$ and provide directions for future mechanistic studies.

\section{Additional files}

Additional file 1: Material and methods. Study groups, experimental protocol, RNA quality control, RNA sequencing quality control and mapping status, and primer sequences. (PDF $395 \mathrm{~kb}$ )

Additional file 2: Hematology and biochemistry after LPS challenge. (PDF $590 \mathrm{~kb}$ )

Additional file 3: List of upregulated and downregulated genes in choroid plexus after LPS challenge. (PDF $458 \mathrm{~kb}$ )

Additional file 4: Gene ontology analyses of DEGs after LPS challenge. (PDF $312 \mathrm{~kb}$ )

Additional file 5: Validation of RNAseq data by qPCR. (PDF $484 \mathrm{~kb}$ )

\section{Abbreviations}

ChP: Choroid plexus; CNS: Central nervous system; DEA: Differential expressed analysis; DEGs: Differentially expressed genes; GO: Gene ontology; IL1R: Interleukin 1 receptor; INF: Interferon; LPS: Lipopolysaccharide; NoRT: No reverse transcriptase; NTC: No template control; qPCR: Quantitative real-time polymerase chain reaction; RIN: RNA integrity number

\section{Acknowledgements}

The authors are grateful to Siri Bjerkreim Hamre and Wenche Okstad for skillful laboratory work. The authors acknowledge Lucy Robertson for proofreading the manuscript.

Funding

The research was supported by The Research Council of Norway.

\section{Availability of data and materials}

Data and material supporting the conclusions are contained within the manuscript.

\section{Authors' contributions}

$C E, M A T, A E, M K B$, and $\varnothing S$ designed the study. $\varnothing S, M R R, C E, A E$, and MAT performed the experiments. ØS carried out the laboratory procedures, performed the statistical analysis, and drafted the manuscript. All authors have critically read and approved the final manuscript.

\section{Competing interests}

The authors declare that the research was conducted in the absence of any commercial or financial relationships that could be construed as a potential conflict of interest.

\section{Ethics approval}

The animal experiment was performed in compliance with the ethical guidelines and approved by the Norwegian Animal Research Authority (ID 5827,6903 , and 7881) with reference to the Norwegian regulation on animal experimentation (FOR-2015-06-18-761).

\section{Publisher's Note}

Springer Nature remains neutral with regard to jurisdictional claims in published maps and institutional affiliations.

Received: 24 February 2017 Accepted: 9 May 2017

Published online: 22 May 2017

\section{References}

1. Linden R, Martins VR, Prado MA, Cammarota M, Izquierdo I, Brentani RR. Physiology of the prion protein. Physiol Rev. 2008;88(2):673-728.

2. Hetz C, Maundrell K, Soto C. Is loss of function of the prion protein the cause of prion disorders? Trends Mol Med. 2003;9(6):237-43.

3. McLennan NF, Rennison KA, Bell JE, Ironside JW. In situ hybridization analysis of PrP mRNA in human CNS tissues. Neuropathol Appl Neurobiol. 2001; 27(5):373-83.

4. Tanji K, Saeki K, Matsumoto Y, Takeda M, Hirasawa K, Doi K, et al. Analysis of PrPc mRNA by in situ hybridization in brain, placenta, uterus and testis of rats. Intervirology. 1995;38(6):309-15.

5. Bakkebø MK, Mouillet-Richard S, Espenes A, Goldmann W, Tatzelt J, Tranulis MA. The cellular prion protein: a player in immunological quiescence. Front Immunol. 2015;6:450.

6. Isaacs JD, Jackson GS, Altmann DM. The role of the cellular prion protein in the immune system. Clin Exp Immunol. 2006;146(1):1-8.

7. Martin GR, Keenan CM, Sharkey KA, Jirik FR. Endogenous prion protein attenuates experimentally induced colitis. Am J Pathol. 2011;179(5):2290-301.

8. Gourdain P, Ballerini C, Nicot AB, Carnaud C. Exacerbation of experimental autoimmune encephalomyelitis in prion protein (PrPc)-null mice: evidence for a critical role of the central nervous system. J Neuroinflammation. 2012;9:25.

9. Steele AD, Zhou Z, Jackson WS, Zhu C, Auluck P, Moskowitz MA, et al. Context dependent neuroprotective properties of prion protein (PrP). Prion. 2009;3(4):240-9.

10. McLennan NF, Brennan PM, McNeill A, Davies I, Fotheringham A, Rennison $\mathrm{KA}$, et al. Prion protein accumulation and neuroprotection in hypoxic brain damage. Am J Pathol. 2004;165(1):227-35.

11. Spudich A, Frigg R, Kilic E, Kilic U, Oesch B, Raeber A, et al. Aggravation of ischemic brain injury by prion protein deficiency: role of ERK-1/-2 and STAT1. Neurobiol Dis. 2005;20(2):442-9.

12. Hoshino S, Inoue K, Yokoyama T, Kobayashi S, Asakura T, Teramoto A, et al. Prions prevent brain damage after experimental brain injury: a preliminary report. Acta Neurochir Suppl. 2003;86:297-9.

13. Liu J, Zhao D, Liu C, Ding T, Yang L, Yin X, et al. Prion Protein participates in the protection of mice from lipopolysaccharide infection by regulating the inflammatory process. J Mol Neurosci. 2014;55:279-87.

14. Park BS, Lee JO. Recognition of lipopolysaccharide pattern by TLR4 complexes. Exp Mol Med. 2013;45:e66.

15. Dantzer R. Cytokine, sickness behavior, and depression. Immunol Allergy Clin North Am. 2009;29(2):247-64.

16. Salvesen $\varnothing$, Reiten MR, Heegaard PM, Tranulis MA, Espenes A, Skovgaard K, et al. Activation of innate immune genes in caprine blood leukocytes after systemic endotoxin challenge. BMC Vet Res. 2016;12(1):241.

17. Banks WA, Robinson SM. Minimal penetration of lipopolysaccharide across the murine blood-brain barrier. Brain Behav Immun. 2010;24(1):102-9. 
18. Bluthé RM, Walter $V$, Parnet $P$, Layé $S$, Lestage J, Verrier $D$, et al. Lipopolysaccharide induces sickness behaviour in rats by a vagal mediated mechanism. C R Acad Sci III. 1994;317(6):499-503.

19. Bluthé RM, Michaud B, Kelley KW, Dantzer R. Vagotomy blocks behavioural effects of interleukin-1 injected via the intraperitoneal route but not via other systemic routes. Neuroreport. 1996;7(15-17):2823-7.

20. Marques F, Sousa JC, Coppola G, Falcao AM, Rodrigues AJ, Geschwind $\mathrm{DH}$, et al. Kinetic profile of the transcriptome changes induced in the choroid plexus by peripheral inflammation. J Cereb Blood Flow Metab. 2009;29(5):921-32

21. Marques F, Sousa JC, Coppola G, Geschwind DH, Sousa N, Palha JA, et al. The choroid plexus response to a repeated peripheral inflammatory stimulus. BMC Neurosci. 2009;10:135.

22. Schmitt C, Strazielle N, Ghersi-Egea JF. Brain leukocyte infiltration initiated by peripheral inflammation or experimental autoimmune encephalomyelitis occurs through pathways connected to the CSF-filled compartments of the forebrain and midbrain. J Neuroinflammation. 2012;9:187.

23. Marques F, Mesquita SD, Sousa JC, Coppola G, Gao F, Geschwind DH, et al. Lipocalin 2 is present in the EAE brain and is modulated by natalizumab. Front Cell Neurosci. 2012;6:33

24. Czerniawski J, Guzowski JF. Acute neuroinflammation impairs context discrimination memory and disrupts pattern separation processes in hippocampus. J Neurosci. 2014;34(37):12470-80.

25. Shaw KN, Commins S, O'Mara SM. Lipopolysaccharide causes deficits in spatial learning in the watermaze but not in BDNF expression in the rat dentate gyrus. Behav Brain Res. 2001;124(1):47-54.

26. Friedman WJ. Cytokines regulate expression of the type 1 interleukin-1 receptor in rat hippocampal neurons and glia. Exp Neurol. 2001;168(1):23-31.

27. Benestad SL, Austbø L, Tranulis MA, Espenes A, Olsaker I. Healthy goats naturally devoid of prion protein. Vet Res. 2012;43:87.

28. Reiten MR, Bakkebø MK, Brun-Hansen H, Lewandowska-Sabat AM, Olsaker I, Tranulis MA, et al. Hematological shift in goat kids naturally devoid of prion protein. Frontiers in cell and developmental biology. 2015;3:44.

29. Büeler H, Aguzzi A, Sailer A, Greiner RA, Autenried P, Aguet M, et al. Mice devoid of PrP are resistant to scrapie. Cell. 1993;73(7):1339-47.

30. Richt JA, Kasinathan P, Hamir AN, Castilla J, Sathiyaseelan T, Vargas F, et al. Production of cattle lacking prion protein. Nat Biotechnol. 2007;25(1):132-8.

31. Takeuchi Y, Kikusui T, Kizumi O, Ohnishi H, Mori Y. Pathophysiological changes evoked by lipopolysaccharide administration in goats. J Vet Med Sci. 1997;59(2):125-7.

32. Ismail M. A pharmacokinetic study of danofloxacin in febrile goats following repeated administration of endotoxin. J Vet Pharmacol Ther. 2006;29(4):313-6.

33. Mi H, Muruganujan A, Casagrande JT, Thomas PD. Large-scale gene function analysis with the PANTHER classification system. Nat Protoc. 2013; 8(8):1551-66.

34. Gene Ontology Consortium enrichment analysis. http://geneontology.org/. Accessed 11 Dec 2016

35. Livak KJ, Schmittgen TD. Analysis of relative gene expression data using real-time quantitative PCR and the 2(-Delta Delta C(T)) Method. Methods. 2001;25(4):402-8.

36. Strom A, Wang GS, Scott FW. Impaired glucose tolerance in mice lacking cellular prion protein. Pancreas. 2011;40(2):229-32.

37. Wopfner F, Weidenhöfer G, Schneider R, von Brunn A, Gilch S, Schwarz TF, et al. Analysis of 27 mammalian and 9 avian PrPs reveals high conservation of flexible regions of the prion protein. J Mol Biol. 1999;289(5):1163-78.

38. Steele AD, Lindquist $S$, Aguzzi $A$. The prion protein knockout mouse: a phenotype under challenge. Prion. 2007;1(2):83-93.

39. Py BF, Gonzalez SF, Long K, Kim MS, Kim YA, Zhu H, et al. Cochlin produced by follicular dendritic cells promotes antibacterial innate immunity. Immunity. 2013;38(5):1063-72.

40. Sanda C, Weitzel P, Tsukahara T, Schaley J, Edenberg HJ, Stephens MA, et al. Differential gene induction by type I and type II interferons and their combination. J Interferon Cytokine Res. 2006;26(7):462-72.

41. Carter SL, Müller M, Manders PM, Campbell IL. Induction of the genes for CxCl9 and CxCl10 is dependent on IFN-gamma but shows differential cellular expression in experimental autoimmune encephalomyelitis and by astrocytes and microglia in vitro. Glia. 2007;55(16):1728-39.

42. Bainbridge J, Walker KB. The normal cellular form of prion protein modulates T cell responses. Immunol Lett. 2005;96(1):147-50.

43. Murray C, Griffin ÉW, O'Loughlin E, Lyons A, Sherwin E, Ahmed S, et al, Interdependent and independent roles of type I interferons and IL-6 in innate immune, neuroinflammatory and sickness behaviour responses to systemic poly I:C. Brain Behav Immun. 2015;48:274-86.

44. Kuwahara C, Takeuchi AM, Nishimura T, Haraguchi K, Kubosaki A, Matsumoto Y, et al. Prions prevent neuronal cell-line death. Nature. 1999; 400(6741):225-6.

45. Bertuchi FR, Bourgeon DM, Landemberger MC, Martins VR, Cerchiaro G. PrPC displays an essential protective role from oxidative stress in an astrocyte cell line derived from PrPC knockout mice. Biochem Biophys Res Commun. 2012;418(1):27-32

46. Bravard A, Auvré F, Fantini D, Bernardino-Sgherri J, Sissoëff L, Daynac M, et al. The prion protein is critical for DNA repair and cell survival after genotoxic stress. Nucleic Acids Res. 2015;43(2):904-16.

47. Gadotti VM, Bonfield SP, Zamponi GW. Depressive-like behaviour of mice lacking cellular prion protein. Behav Brain Res. 2012;227(2):319-23.

48. Beckman D, Santos LE, Americo TA, Ledo JH, de Mello FG, Linden R. Prion protein modulates monoaminergic systems and depressive-like behavior in mice. J Biol Chem. 2015;290(33):20488-98.

49. Martinez FO, Gordon S. The M1 and M2 paradigm of macrophage activation: time for reassessment. F1000Prime Rep. 2014;6:13.

50. Martinez FO, Helming L, Milde R, Varin A, Melgert BN, Draijer C, et al. Genetic programs expressed in resting and IL-4 alternatively activated mouse and human macrophages: similarities and differences. Blood. 2013; 121(9):e57-69.

51. Köhler C. Allograft inflammatory factor-1/lonized calcium-binding adapter molecule 1 is specifically expressed by most subpopulations of macrophages and spermatids in testis. Cell Tissue Res. 2007:330(2):291-302.

52. Colton CA. Immune heterogeneity in neuroinflammation: dendritic cells in the brain. J Neuroimmune Pharmacol. 2013;8(1):145-62.

53. Hasegawa-Ishii S, Inaba M, Umegaki H, Unno K, Wakabayashi K, Shimada A. Endotoxemia-induced cytokine-mediated responses of hippocampal astrocytes transmitted by cells of the brain-immune interface. Sci Rep. 2016;6:25457.

54. Müller M, Carter S, Hofer MJ, Campbell IL. Review: the chemokine receptor CXCR3 and its ligands CXCL9, CXCL10 and CXCL11 in neuroimmunity-a tale of conflict and conundrum. Neuropathol Appl Neurobiol. 2010;36(5):368-87.

55. Pedersen $M \varnothing$, Jensen R, Pedersen DS, Skjolding AD, Hempel C, Maretty L, et al. Metallothionein-I + II in neuroprotection. Biofactors. 2009;35(4):315-25.

56. Brahmachari S, Fung YK, Pahan K. Induction of glial fibrillary acidic protein expression in astrocytes by nitric oxide. J Neurosci. 2006;26(18):4930-9.

57. Hirsch TZ, Hernandez-Rapp J, Martin-Lannerée S, Launay JM, MouilletRichard S. $\operatorname{PrP}(C)$ signalling in neurons: from basics to clinical challenges. Biochimie. 2014;104:2-11.

58. Buttini M, Limonta S, Boddeke HW. Peripheral administration of lipopolysaccharide induces activation of microglial cells in rat brain. Neurochem Int. 1996;29(1):25-35

59. Chen Z, Jalabi W, Shpargel KB, Farabaugh $K T$, Dutta R, Yin $X$, et al. Lipopolysaccharide-induced microglial activation and neuroprotection against experimental brain injury is independent of hematogenous TLR4. J Neurosci. 2012;32(34):11706-15.

60. Mariante RM, Nóbrega A, Martins RA, Areal RB, Bellio M, Linden R. Neuroimmunoendocrine regulation of the prion protein in neutrophils. J Biol Chem. 2012;287(42):35506-15.

61. Wang V, Chuang TC, Hsu YD, Chou WY, Kao MC. Nitric oxide induces prion protein via MEK and p38 MAPK signaling. Biochem Biophys Res Commun. 2005;333(1):95-100.

62. Brogna S, Wen J. Nonsense-mediated mRNA decay (NMD) mechanisms. Nat Struct Mol Biol. 2009;16(2):107-13. 